\title{
FIXED POINT AND CONTINUATION RESULTS FOR CONTRACTIONS IN METRIC AND GAUGE SPACES
}

\author{
M. FRIGON \\ Département de mathématiques et de statistique, Université de Montréal \\ C.P. 6128, succ. Centre-Ville, Montréal (Québec), H3C 3J7, Canada \\ E-mail: frigon@dms.umontreal.ca
}

\begin{abstract}
We present an overview of generalizations of Banach's fixed point theorem and continuation results for contractions, i.e., results establishing that the existence of a fixed point is preserved by suitable homotopies. We will consider single-valued and multi-valued contractions in metric and in gauge spaces.
\end{abstract}

0. Introduction. We present an overview of fixed point results for contractions in metric and in gauge spaces. The first result is the famous contraction principle due to Banach [4]. Weakening the contraction condition permitted many authors to generalize the Banach fixed point theorem, see $[6,7,8,14,15,16,27,40,50,51,60]$. Banach's fixed point theorem was also generalized to locally convex spaces by Cain and Nashed [9], and to uniform spaces by Knill [38]. See also [20, 23, 28, 62] for results in Fréchet or gauge spaces.

The question of the convergence of a sequence of fixed points of a converging sequence of contractions is then raised. An affirmative answer to this question was obtained by Bonsall [5] for a sequence of contractions $\left\{f_{n}\right\}$ converging pointwise to $f_{0}$ when the constants of contraction are the same for every $f_{n}$. This result was extended by Reich [51] for more general contractions. Moreover, the constants of contraction may vary with $n$ if stronger assumptions are imposed on the space or if the convergence of $\left\{f_{n}\right\}$ is uniform, see Nadler [42].

One could also ask if one can replace the sequence of contractions by a family of contractions or homotopies of contractions. We present an overview of continuation results for homotopies of contractions on metric or gauge spaces $h: X \times[0,1] \rightarrow E$ with $X$ a closed subset of $E$. More precisely, we give conditions which ensure that if $h(\cdot, 0)$ has a fixed point then $h(\cdot, t)$ has a fixed point for every $t \in[0,1]$. Usually the space $X$ is

2000 Mathematics Subject Classification: Primary 47H10; Secondary 47H04, 47H09.

Key words and phrases: fixed point theory, continuation results, homotopic invariance, singlevalued and multi-valued contractions, metric space, gauge space.

This work was partially supported by CRSNG Canada

The paper is in final form and no version of it will be published elsewhere. 
the closure of an open set. However, as it was explained in [20], potential applications in gauge spaces lead to consider homotopies defined on a subset $X$ with empty interior. For this reason, the usual condition $x \neq h(x, t)$ for $x \in \partial X$ should be replaced, and a more general notion of contraction is suitable, see [20].

We also present some fixed point and continuation results for multi-valued contractions in metric and gauge spaces. The first fixed point result for multi-valued contraction was obtained by Nadler [43], while the first continuation result is due to Frigon and Granas [22]; see [12, 19, 25, 39, 44, 48, 61] for generalizations and related results.

Obviously, this overview on fixed point and continuation results for contractions cannot be complete but it gives a good idea of results which can be found in the literature. The reader is referred to $[32,29,30,36,37]$ and the references therein for more results on contractive and non-expansive mappings.

The paper is divided into six parts: (1) single-valued contractions in metric spaces; (2) continuation results for single-valued contractions in metric spaces; (3) multi-valued contractions in metric spaces; (4) continuation results for multi-valued contractions in metric spaces; (5) single-valued contractions in gauge spaces; (6) multi-valued contractions in gauge spaces.

\section{Single-valued contractions}

1.1. Existence results. The most famous fixed point theorem is certainly the contraction principle established by Banach in 1922 [4]. This result establishing the existence of a fixed point of a contraction defined on a Banach space was very useful in particular in the theory of differential and integral equations, in the theory of chaos and in numerical analysis.

Theorem 1.1 (Banach, 1922). Let $(E,\|\cdot\|)$ be a Banach space and $f: E \rightarrow E$ a contraction, i.e.,

there exists $k<1$ such that $\|f(x)-f(y)\| \leq k\|x-y\|$ for every $x, y \in E$.

Then $f$ has a fixed point $x_{0}$.

Caccioppoli [8] formulated this result in complete metric space. He also observed that it is clear from the proof that the fixed point $x_{0}$ is unique and $f^{n}(x) \rightarrow x_{0}$ for every $x \in E$.

Many generalizations of this principle were given with the condition of contraction replaced by a weaker one. We state a result of Weissinger [63].

Theorem 1.2 (Weissinger, 1952). Let $(E, d)$ be a complete metric space and $f: E \rightarrow E$ such that for every $n \in \mathbb{N}$, there exists $k_{n}$ such that

$$
d\left(f^{n}(x), f^{n}(y)\right) \leq k_{n} d(x, y), \text { and } \sum_{n=1}^{\infty} k_{n}<\infty .
$$

Then $f$ has a unique fixed point $x_{0}$, which is such that $f^{n}(x) \rightarrow x_{0}$ for every $x \in E$. Moreover,

$$
d\left(x_{0}, f^{n}(x)\right) \leq d\left(f^{m}(x), f^{m+1}(x)\right) \sum_{i=n-m}^{\infty} k_{i} \quad \forall m \in\{0,1, \ldots, n-1\} .
$$


It was natural to ask if the Banach contraction principle holds when $k=1$ and the inequality replaced by a strict inequality, i.e., if $d(f(x), f(y))<d(x, y)$ for all $x \neq y$. This is not possible as it is shown in the following example.

EXAMPLE 1.3. Let $E=l^{1}$ be the space of real sequences $x=\left(x_{n}\right)_{n \in \mathbb{N}}$ endowed with the usual norm $\|x\|_{1}=\sum_{n}\left|x_{n}\right|$. Let $f: l^{1} \rightarrow l^{1}$ be defined by

$$
f\left(x_{1}, x_{2}, \ldots\right)=\left(1, \frac{x_{1}}{2}, \frac{2 x_{2}}{3}, \frac{3 x_{3}}{4}, \ldots\right) .
$$

It is clear that $\|f(x)-f(y)\|_{1}<\|x-y\|_{1}$ for all $x \neq y$ in $l^{1}$. However, $x=f(x)$ if and only if $x=\left(1, \frac{1}{2}, \frac{1}{3}, \ldots\right)$ but $x \notin l^{1}$. So $f$ has no fixed point.

Therefore, with such a weak condition of contraction, extra assumptions on the space are needed as was shown by Edelstein [15], [16].

TheOrEm 1.4 (Edelstein, 1961-62). Let $(E, d)$ be a complete metric space, $f: E \rightarrow E$. (a) If $d(f(x), f(y))<d(x, y)$ for all $x, y \in E$, and if there exists $x_{1}$ such that $\left\{f^{n}\left(x_{1}\right)\right\}$ has a subsequence converging to $x_{0}$, then $x_{0}$ is the unique fixed point of $f$. (Condition satisfied if $E$ is compact).

(b) If $f$ is $(\varepsilon, k)$-uniformly locally contractive, i.e., there exist $\varepsilon>0$ and $k<1$ such that

$$
d(x, y)<\varepsilon \Longrightarrow d(f(x), f(y)) \leq k d(x, y),
$$

and if $E$ is $\varepsilon$-chainable (i.e., for every $x, y \in E$, there exist $x_{0}, \ldots, x_{n}$ such that $x=x_{0}$, $y=x_{n}$ and $d\left(x_{k-1}, x_{k}\right)<\varepsilon$ for all $\left.k=1, \ldots, n\right)$, then $f$ has a unique fixed point.

A simple proof of Theorem 1.4(a) can be found in [30].

From the previous example and theorem, one sees that to obtain generalizations of the Banach contraction principle in complete metric spaces, one should look for a condition weaker than

$$
\exists k \in[0,1) \text { such that } d(f(x), f(y)) \leq k d(x, y) \quad \forall x, y \in E ;
$$

and stronger than

$$
d(f(x), f(y))<d(x, y) \quad \forall x \neq y \in E .
$$

This was done in particular by Rakotch [50], Browder [7], Boyd and Wong [6], Geraghty [27] and Matkowski [40].

THEOREM 1.5. Let $(E, d)$ be a complete metric space and $f: E \rightarrow E$ such that there exists $\psi:[0, \infty) \rightarrow[0, \infty)$ with

$$
d(f(x), f(y)) \leq \psi(d(x, y)) \quad \text { for every } x, y \in E .
$$

Assume one of the following conditions is satisfied:

(a) [Rakotch, 1962] $\psi(t)=t \phi(t)$ with $\phi:(0, \infty) \rightarrow[0,1)$ decreasing.

(b) [Browder, 1968] $\psi$ is non-decreasing and continuous from the right such that

$$
\psi(t)<t \quad \text { for every } t>0 .
$$

(c) [Boyd and Wong, 1969] $\psi$ is upper semi-continuous from the right or $\lim \sup \psi(s)<t$, and such that

$$
\psi(t)<t \quad \text { for every } t>0 \text {. }
$$


(d) [Geraghty, 1973] $\psi(t)=t \phi(t)$ with $\phi:[0, \infty) \rightarrow[0,1)$ such that

$$
\phi\left(t_{n}\right) \rightarrow 1 \Longrightarrow t_{n} \rightarrow 0 .
$$

(e) [Matkowski, 1994] $\psi$ is continuous at 0 , subadditive and there exists $\left\{t_{n}\right\}$ converging to 0 such that

$$
\psi\left(t_{n}\right)<t_{n} \quad \text { for every } n \in \mathbb{N} \text {. }
$$

Then $f^{n}(x) \rightarrow x_{0}=f\left(x_{0}\right)$ for every $x \in E$.

Idea of the proof. Take $x \in E$. Using the fact that

$$
d\left(f^{n}(x), f^{n+1}(x)\right) \leq \psi\left(d\left(f^{n-1}(x), f^{n}(x)\right)\right)
$$

and the properties of $\psi$, deduce that

$$
\lim _{n \rightarrow \infty} d\left(f^{n}(x), f^{n+1}(x)\right)=0 .
$$

Then show that $\left\{f^{n}(x)\right\}$ is a Cauchy sequence converging to $x_{0}$. Finally, conclude that $x_{0}$ is the unique fixed point of $f$.

The reader is referred to [35] for a comparison of the previous conditions. Also, it is worth to mention that most of non-expansive mappings (in the sense of Baire category) satisfy Rakotch's condition (Theorem 1.5(a)), see [58, 59].

Some of the previous conditions can be weakened if one assumes that $E$ is metrically convex, i.e.,

$$
\forall x \neq y, \exists z \in E \quad \text { such that } \quad d(x, y)=d(x, z)+d(z, y) .
$$

TheOREM 1.6. Let $(E, d)$ be a metrically convex complete metric space and $f: E \rightarrow E$ such that there exists $\psi:[0, \infty) \rightarrow[0, \infty)$ with

$$
d(f(x), f(y)) \leq \psi(d(x, y)) \quad \text { for every } x, y \in E .
$$

Assume one of the following conditions is satisfied:

(a) [Boyd and Wong, 1969] $\psi$ satisfies

$$
\psi(t)<t \quad \text { for every } t>0 .
$$

(b) [Matkowski, 1994] $\psi$ is continuous at 0 , and there exists $\left\{t_{n}\right\}$ converging to 0 such that

$$
\psi\left(t_{n}\right)<t_{n} \quad \text { for every } n \in \mathbb{N} \text {. }
$$

Then $f^{n}(x) \rightarrow x_{0}=f\left(x_{0}\right)$ for every $x \in E$.

In the two previous results $\psi$ depended on $d(x, y)$, Dugundji and Granas [14] considered the case of a function depending on $x, y$.

Theorem 1.7 (Dugundji and Granas, 1978). Let $(E, d)$ be a complete metric space and $f: E \rightarrow E$ such that there exists $\theta: E \times E \rightarrow(0, \infty)$ with

$$
d(f(x), f(y)) \leq d(x, y)-\theta(x, y) \quad \text { for every } x \neq y
$$

and

$$
\inf \{\theta(x, y): a \leq d(x, y) \leq b\}>0 \quad \text { for every } b \geq a>0 .
$$

Then $f^{n}(x) \rightarrow \hat{x}=f(\hat{x})$ for every $x \in E$. 
Proof. Take $x_{0} \in E$ and define inductively $x_{n}=f^{n}\left(x_{0}\right)$. Observe that

$$
d\left(x_{2}, x_{1}\right) \leq d\left(x_{1}, x_{0}\right)-\theta\left(x_{1}, x_{0}\right) .
$$

This implies that

$$
d\left(x_{3}, x_{2}\right) \leq d\left(x_{2}, x_{1}\right)-\theta\left(x_{2}, x_{1}\right) \leq d\left(x_{1}, x_{0}\right)-\theta\left(x_{1}, x_{0}\right)-\theta\left(x_{2}, x_{1}\right) .
$$

Repeating this argument, we deduce that

$$
d\left(x_{n}, x_{n+1}\right) \leq d\left(x_{0}, x_{1}\right)-\sum_{j=1}^{n} \theta\left(x_{j-1}, x_{j}\right) .
$$

This inequality implies that the series $\sum_{j=1}^{\infty} \theta\left(x_{j-1}, x_{j}\right)$ is convergent.

For $0<a \leq b$, define

$$
\mu(a, b)=\inf \{\theta(x, y): a \leq d(x, y) \leq b\} \quad \text { and } \quad \gamma(a, b)=\min \{a, \mu(a, b)\} .
$$

Let $\varepsilon \in\left(0, d\left(x_{0}, x_{1}\right)\right)$, and choose $N \in \mathbb{N}$ such that

$$
\theta\left(x_{n}, x_{n+1}\right)<\mu\left(\gamma(\varepsilon / 2, \varepsilon), d\left(x_{0}, x_{1}\right)\right) \quad \text { for every } n \geq N .
$$

This, and the fact that $d\left(x_{N}, x_{N+1}\right) \leq d\left(x_{0}, x_{1}\right)$ imply that

$$
d\left(x_{N}, x_{N+1}\right)<\gamma(\varepsilon / 2, \varepsilon) .
$$

Observe that

$$
\begin{aligned}
d\left(x_{N+2}, x_{N}\right) & \leq d\left(x_{N+2}, x_{N+1}\right)+d\left(x_{N+1}, x_{N}\right) \\
& \leq 2 d\left(x_{N+1}, x_{N}\right)-\theta\left(x_{N+1}, x_{N}\right)<\varepsilon
\end{aligned}
$$

Similarly,

$$
\begin{aligned}
d\left(x_{N+3}, x_{N}\right) & \leq d\left(x_{N+3}, x_{N+1}\right)+d\left(x_{N+1}, x_{N}\right) \\
& \leq d\left(x_{N+2}, x_{N}\right)-\theta\left(x_{N+2}, x_{N}\right)+d\left(x_{N+1}, x_{N}\right) \\
& <d\left(x_{N+2}, x_{N}\right)-\theta\left(x_{N+2}, x_{N}\right)+\gamma(\varepsilon / 2, \varepsilon) \leq \varepsilon
\end{aligned}
$$

Repeating this argument implies that $x_{n} \in B\left(x_{N}, \varepsilon\right)$ for all $n \geq N$. So,

$$
d\left(x_{n}, x_{m}\right)<2 \varepsilon \text { for every } n, m \geq N .
$$

Since $\varepsilon$ is arbitrary, $\left\{x_{n}\right\}$ is a Cauchy sequence, and hence converges to $x \in E$. From the continuity of $f$, we deduce that $x$ is a fixed point of $f$. The uniqueness of the fixed point is obvious.

In [51], Reich generalized the contraction condition to the following one:

there exist $k, l, m \in[0,1)$ such that $k+l+m<1$ and

$$
d(f(x), f(y)) \leq k d(x, f(x))+l d(y, f(y))+m d(x, y) \quad \text { for every } x, y \in E .
$$

This condition was also generalized by many authors, see [60] for a comparison between different conditions. 
1.2. Stability results. Now, we are interested in the behavior of a sequence $\left\{x_{n}\right\}$ such that $x_{n}$ is the unique fixed point of the contraction $f_{n}$, and $\left\{f_{n}\right\}$ converges to $f_{0}$. It was shown by Bonsall [5], and then generalized by Reich [51], that if the constant of contraction is the same for all $n$ then the pointwise convergence is sufficient to guarantee that $x_{n} \rightarrow x_{0}$. However, Nadler [42] established an analogous result when the convergence of $\left\{f_{n}\right\}$ is uniform and the constant of contraction varies with $n$.

Theorem 1.8. Let $(E, d)$ be a complete metric space, and $f_{n}: E \rightarrow E$ for $n \in \mathbb{N} \cup\{0\}$. Assume that one of the following conditions is true.

(a) [Bonsall, 1962] $f_{n} \rightarrow f_{0}$ pointwise and there exists $k<1$ such that for every $n \in \mathbb{N} \cup\{0\}$

$$
d\left(f_{n}(x), f_{n}(y)\right) \leq k d(x, y) \quad \text { for every } x, y \in E .
$$

(b) [Nadler, 1968] For every $n \in \mathbb{N} \cup\{0\}$, there exists $k_{n}<1$ such that

$$
d\left(f_{n}(x), f_{n}(y)\right) \leq k_{n} d(x, y) \quad \text { for every } x, y \in E,
$$

and $f_{n} \rightarrow f_{0}$ uniformly, or $f_{n} \rightarrow f_{0}$ pointwise and $E$ is locally compact.

(c) [Reich, 1971] $f_{n} \rightarrow f_{0}$ pointwise and there exist $k, l, m \geq 0$ such that $k+l+m<1$, and for all $n \in \mathbb{N} \cup\{0\}$,

$$
d\left(f_{n}(x), f_{n}(y)\right) \leq k d(x, y)+l d\left(x, f_{n}(x)\right)+m d\left(y, f_{n}(y)\right) \quad \text { for every } x, y \in E .
$$

Then $x_{n}=f_{n}\left(x_{n}\right) \rightarrow x_{0}=f_{0}\left(x_{0}\right)$.

Proof. (a) Let $x_{n}=f_{n}\left(x_{n}\right)$ for $n \in \mathbb{N} \cup\{0\}$. Then,

$$
\begin{aligned}
d\left(x_{n}, x_{0}\right) & =d\left(f_{n}\left(x_{n}\right), f_{0}\left(x_{0}\right)\right) \\
& \leq d\left(f_{n}\left(x_{n}\right), f_{n}\left(x_{0}\right)\right)+d\left(f_{n}\left(x_{0}\right), f_{0}\left(x_{0}\right)\right) \\
& \leq k d\left(x_{n}, x_{0}\right)+d\left(f_{n}\left(x_{0}\right), f_{0}\left(x_{0}\right)\right) .
\end{aligned}
$$

Therefore, $x_{n} \rightarrow x_{0}$. The statement (c) can be proved similarly.

(b) Again $x_{n}=f_{n}\left(x_{n}\right)$. If $f_{n} \rightarrow f_{0}$ uniformly, then

$$
\begin{aligned}
d\left(x_{n}, x_{0}\right) & =d\left(f_{n}\left(x_{n}\right), f_{0}\left(x_{0}\right)\right) \\
& \leq d\left(f_{n}\left(x_{n}\right), f_{0}\left(x_{n}\right)\right)+d\left(f_{0}\left(x_{n}\right), f_{0}\left(x_{0}\right)\right) \\
& \leq k_{0} d\left(x_{n}, x_{0}\right)+d\left(f_{n}\left(x_{n}\right), f_{0}\left(x_{n}\right)\right) .
\end{aligned}
$$

Therefore, $x_{n} \rightarrow x_{0}$.

Now assume that $f_{n} \rightarrow f_{0}$ pointwise and $E$ is locally compact. Let $\varepsilon>0$ be sufficiently small such that $K:=\overline{B\left(x_{0}, \varepsilon\right)}$ is compact. Since the sequence $\left\{f_{n}\right\}$ is equicontinuous and converges pointwise to $f_{0}$ on the compact set $K$, it converges uniformly on $K$ to $f_{0}$. Fix $N \in \mathbb{N}$ such that for all $n \geq N$,

$$
d\left(f_{n}(x), f_{0}(x)\right) \leq\left(1-k_{0}\right) \varepsilon \quad \text { for every } x \in K .
$$

It follows that for every $x \in K$,

$$
\begin{aligned}
d\left(f_{n}(x), x_{0}\right) & \leq d\left(f_{n}(x), f_{0}(x)\right)+d\left(f_{0}(x), f_{0}\left(x_{0}\right)\right) \\
& \leq\left(1-k_{0}\right) \varepsilon+k_{0} d\left(x, x_{0}\right) \leq \varepsilon .
\end{aligned}
$$


This shows that $f_{n}$ maps $K$ in $K$ and hence has a fixed point in $K$. The uniqueness of the fixed point implies that $x_{n} \in K$. Since we can choose $\varepsilon$ arbitrarily small, we deduce that $x_{n} \rightarrow x_{0}$.

2. Continuation results for single-valued contractions. In this section, we study continuation results. More precisely, we are interested in conditions under which a family of contractions $\left\{f_{\lambda}\right\}$ has a fixed point for each value of the parameter $\lambda$. It is also interesting to study the properties of $\lambda \mapsto x_{\lambda}=f_{\lambda}\left(x_{\lambda}\right)$. In 1969, Nussbaum [45] extended a continuation result for compact maps to $k$-set contractions.

TheOrem 2.1 (Nussbaum, 1969). Let $E$ be a Banach space, $U$ bounded and open in $E$ with $0 \in U$, and $f: \bar{U} \rightarrow E$ a $k$-set contraction for $k<1$ (i.e., $\alpha(f(A)) \leq k \alpha(A)$ for all bounded subset $A$, where $\alpha$ is the Kuratowski measure of non-compactness). Assume that

$$
x=\lambda f(x) \text { for } x \in \partial U \Longrightarrow \lambda \geq 1 \text {. }
$$

Then $f$ has a fixed point in $\bar{U}$.

An elementary proof of this result was given by Gatica and Kirk [26] in the case where $f$ is a contraction. They removed the assumption that $U$ is bounded. In [24], Granas, Guennoun and myself showed that a Lipschitzian curve of fixed point $\lambda \mapsto x_{\lambda}=\lambda f\left(x_{\lambda}\right)$ is obtained for $\lambda \in[0,1]$.

TheOREM 2.2. Let $E$ be a Banach space, $Y$ closed and convex in $E, U$ open in $Y$ with $0 \in U$, and $f: \bar{U} \rightarrow Y$ a contraction. Assume that

$$
x=\lambda f(x) \text { for } x \in \partial_{Y} U \Longrightarrow \lambda \geq 1 \text {. }
$$

Then $f$ has a fixed point in $\bar{U}$. Moreover, there is a Lipschitzian curve $\lambda \mapsto x_{\lambda}=\lambda f\left(x_{\lambda}\right)$ defined on $[0,1]$.

Proof. First of all, notice that if $x=\lambda f(x)$ for $\lambda \in[0,1]$, then

$$
\|x\|=\lambda\|f(x)\| \leq \lambda\|f(x)-f(0)\|+\|f(0)\| \leq k\|x\|+\|f(0)\|,
$$

and

$$
\|f(x)\| \leq k\|x\|+\|f(0)\|
$$

where $k$ is the constant of contraction. So, there exists $M>0$ such that $\|f(x)\| \leq M$ for all $x=\lambda f(x)$ for some $\lambda \in[0,1]$.

Let $Q=\{\lambda \in[0,1): \lambda f$ has a fixed point $\}$. Observe that $0 \in Q$. Let $\lambda_{0} \in Q$ and $x_{0}=$ $\lambda_{0} f\left(x_{0}\right)$. By assumption, $x_{0} \notin \partial_{Y} U$. So, there exists $s>0$ such that $\overline{B\left(x_{0}, s\right)} \cap Y \subset \bar{U}$. For $\lambda \in[0,1)$ such that $\left|\lambda-\lambda_{0}\right|\left\|f\left(x_{0}\right)\right\|<s(1-k), \lambda f: \overline{B\left(x_{0}, s\right)} \cap Y \rightarrow \overline{B\left(x_{0}, s\right)} \cap Y$. Indeed, for $x \in \overline{B\left(x_{0}, s\right)} \cap Y$,

$$
\left\|x_{0}-\lambda f(x)\right\| \leq\left\|\lambda_{0} f\left(x_{0}\right)-\lambda f\left(x_{0}\right)\right\|+\lambda\left\|f\left(x_{0}\right)-f(x)\right\| \leq s(1-k)+k\left\|x_{0}-x\right\| \leq s .
$$

The Banach contraction principle implies that $\lambda \in Q$ and hence $Q$ is open.

Now, we claim that $Q$ is closed. Indeed, let $\lambda_{1}, \lambda_{2} \in Q$ and $x_{1}=\lambda_{1} f\left(x_{1}\right), x_{2}=$ $\lambda_{2} f\left(x_{2}\right)$. We have

$$
\left\|x_{1}-x_{2}\right\| \leq\left\|\lambda_{1} f\left(x_{1}\right)-\lambda_{2} f\left(x_{1}\right)\right\|+\lambda_{2}\left\|f\left(x_{1}\right)-f\left(x_{2}\right)\right\| \leq M\left|\lambda_{1}-\lambda_{2}\right|+k\left\|x_{1}-x_{2}\right\|,
$$


and hence,

$$
\left\|x_{1}-x_{2}\right\| \leq \frac{M}{1-k}\left|\lambda_{1}-\lambda_{2}\right|
$$

It follows that $Q=[0,1)$ since $[0,1)$ is connected. Moreover, inequality $(2.1)$ implies the existence of $\lambda \mapsto x_{\lambda}=\lambda f\left(x_{\lambda}\right)$ a Lipschitzian curve on $[0,1]$, with $x_{1} \in \bar{U}$.

Using the same arguments, Granas [31] extended this result to more general homotopies in complete metric spaces. Note that condition (ii) of the following result can be generalized in order to keep the curve $t \mapsto x_{t}$ continuous, see Precup's result [48].

Theorem 2.3 (Granas, 1994). Let $E$ be a complete metric space, $U \subset E$ open, and $h: \bar{U} \times[0,1] \rightarrow E$ such that

(i) there exists $k<1$ such that $d(h(x, t), h(y, t)) \leq k d(x, y)$ for every $x, y \in E$ and every $t \in[0,1]$;

(ii) there exists $M \geq 0$ such that $d(h(x, t), h(x, s)) \leq M|t-s|$ for every $x \in E$ and every $s, t \in[0,1]$;

(iii) $x \neq h(x, t)$ for $x \in \partial U$, and $t \in[0,1]$.

If $h(\cdot, 0)$ has a fixed point, then there exists a Lipschitzian curve $t \mapsto x_{t}=h\left(x_{t}, t\right)$ defined on $[0,1]$.

This type of result is also true with the notion of contraction introduced by Dugundji and Granas as shown in [19].

Theorem 2.4 (Frigon, 1996). Let $E$ be a complete metric space, $U \subset E$ open, and $h: \bar{U} \times[0,1] \rightarrow E$ such that

(i) there exists $\theta: E \times E \rightarrow(0, \infty)$ such that

$$
d(h(x, t), h(y, t)) \leq d(x, y)-\theta(x, y) \quad \text { for every } x \neq y \text { and } t \in[0,1],
$$

and

$$
\inf \{\theta(x, y): d(x, y) \geq a\}>0 \quad \text { for every } a>0
$$

(ii) there exists a continuous function $\phi:[0,1] \rightarrow \mathbb{R}$ such that for every $x \in \bar{U}$, and $t, s \in[0,1], d(h(x, t), h(x, s)) \leq|\phi(t)-\phi(s)|$;

(iii) $x \neq h(x, t)$ for $x \in \partial U$, and $t \in[0,1]$.

If $h(\cdot, 0)$ has a fixed point, then $h(\cdot, t)$ has a fixed point for every $t \in[0,1]$.

Proof. For $0<a \leq b$, let

$$
\mu(a, b)=\inf \{\theta(x, y): a \leq d(x, y) \leq b\} \quad \text { and } \quad \gamma(a, b)=\min \{a, \mu(a, b)\} .
$$

Define

$$
Q=\{\lambda \in[0,1]: h(\cdot, \lambda) \text { has a fixed point }\} .
$$

To show that $Q$ is open, take $\lambda_{0} \in Q$ and $x=h\left(x, \lambda_{0}\right)$. Let $r>0$ be such that $B(x, r) \subset U$, and let $\delta>0$ such that for $\left|\lambda-\lambda_{0}\right|<\delta,\left|\phi(\lambda)-\phi\left(\lambda_{0}\right)\right|<\gamma(r / 2, r)$. Then

$$
\begin{aligned}
d(x, h(x, \lambda)) & \leq d\left(x, h\left(x, \lambda_{0}\right)\right)+d\left(h\left(x, \lambda_{0}\right), h(x, \lambda)\right) \\
& \leq\left|\phi(\lambda)-\phi\left(\lambda_{0}\right)\right|<\gamma(r / 2, r) .
\end{aligned}
$$


By arguing as in Theorem 1.7, it can be shown that $h(\cdot, \lambda)$ has a fixed point in $\overline{B(x, r)}$ for every $\lambda$ such that $\left|\lambda-\lambda_{0}\right|<\delta$.

To show that $Q$ is closed, take $\left\{\lambda_{n}\right\}$ in $Q$ such that $\lambda_{n} \rightarrow \lambda$. Let $x_{n}=h\left(x_{n}, \lambda_{n}\right)$. Fix $\varepsilon>0$. Define $k=\inf \{\mu(\varepsilon, b): b \geq \varepsilon\}$.

Let $N \in \mathbb{N}$ be such that for all $n, m \geq N,\left|\phi\left(\lambda_{n}\right)-\phi\left(\lambda_{m}\right)\right|<k$. Then $d\left(x_{n}, x_{m}\right)<\varepsilon$ for every $n, m \geq N$. Indeed, otherwise,

$$
\begin{aligned}
d\left(x_{n}, x_{m}\right) & \leq d\left(h\left(x_{n}, \lambda_{n}\right), h\left(x_{n}, \lambda_{m}\right)\right)+d\left(h\left(x_{n}, \lambda_{m}\right), h\left(x_{m}, \lambda_{m}\right)\right) \\
& \leq\left|\phi\left(\lambda_{n}\right)-\phi\left(\lambda_{m}\right)\right|+d\left(x_{n}, x_{m}\right)-\theta\left(x_{n}, x_{m}\right) \\
& <k+d\left(x_{n}, x_{m}\right)-\theta\left(x_{n}, x_{m}\right) \leq d\left(x_{n}, x_{m}\right),
\end{aligned}
$$

which is a contradiction. So $Q$ is closed.

Therefore, if $h(\cdot, 0)$ has a fixed point, $Q=[0,1]$, so $h(\cdot, t)$ has a fixed point.

Condition (i) of Theorem 2.3 can also be weakened by considering different constants of contraction. In this case, a stronger assumption on the space is needed. Also, [0, 1] can be replaced by a metric space as shown in [34] for $U=E$. Notice that in the following result the family of maps $\{h(\cdot, \lambda)\}$ is defined on the whole space.

THEOREM 2.5 (Jachymski, 1996). Let E be a locally compact complete metric space, $(M, \rho)$ a metric space and $h: E \times M \rightarrow E$ such that

(i) for every $\lambda \in M, x \mapsto h(x, \lambda)$ is a contraction (not necessarily with the same constant);

(ii) for every $x \in E, \lambda \mapsto h(x, \lambda)$ is continuous.

Then there exists a continuous curve $\lambda \mapsto x_{\lambda}=h\left(x_{\lambda}, \lambda\right)$ defined on $M$.

Using this theorem, Jachymski obtained a continuation result for attractors of iterated function systems.

Definition 2.6. Let $f_{1}, \ldots, f_{n}: E \rightarrow E$ be contractions. Then $\left((E, d), f_{1}, \ldots f_{n}\right)$ is called a hyperbolic iterated function system (IFS). It generates a mapping $\hat{f}: K(E) \rightarrow$ $K(E)$ defined by

$$
\hat{f}(X)=\bigcup_{i=1}^{n} f_{i}(X),
$$

where $K(E)$ is the space of compact subsets of $E$ endowed with the Hausdorff metric. It can be shown that $\hat{f}$ is a contraction. The fixed point of $\hat{f}$ is denoted by $A$ and is called the attractor of the IFS.

Theorem 2.7 (Jachymski, 1996). Let $E$ be a locally compact complete metric space, $(M, \rho)$ a metric space, and for each $\lambda \in M,\left((E, d), f_{1}(\cdot, \lambda), \ldots f_{n}(\cdot, \lambda)\right)$ an IFS such that for $i=1, \ldots, n, f_{i}$ is continuous with respect to its second variable. Then the attractor $A(\lambda)$ depends continuously on the parameter $\lambda \in M$.

Here is a result established by Chiş and Precup [11] for a homotopy defined on an arbitrary closed set $X$ of $E$. Of course, the condition on $\partial X$ had to be changed. 
Theorem 2.8 (Chiş and Precup, 2004). Let $E$ be a complete metric space, $X \subset E$ closed, and $h: X \times[0,1] \rightarrow E$ such that

(i) there exist $k>0, l \geq 0$ such that $k+2 l<1$, and for every $x, y \in X$ and $t \in[0,1]$,

$$
d(h(x, t), h(y, t)) \leq k d(x, y)+l(d(x, h(x, t))+d(y, h(y, t)))
$$

(ii) $t \mapsto h(x, t)$ is continuous uniformly on $X$;

(iii) $\inf \{d(x, y): x=h(x, t), y \in E \backslash X\}>0$.

If $h(\cdot, 0)$ has a fixed point, then $h(\cdot, t)$ has a unique fixed point for each $t \in[0,1]$.

Precup [49], and with O'Regan [46] obtained continuation results with two metrics on $E$ and also with generalized metrics.

\section{Multi-valued contractions}

3.1. Existence results. In this section, we present fixed point results for multi-valued contractions. Let $(E, d)$ be a complete metric space. We denote by $D$ the generalized Hausdorff metric, i.e., for $X, Y$ closed subsets of $E$,

$$
D(X, Y)=\inf \{\varepsilon>0: \varepsilon \in \mathcal{E}(X, Y)\},
$$

where

$$
\mathcal{E}(X, Y)=\{\varepsilon>0: X \subset B(Y, \varepsilon), Y \subset B(X, \varepsilon)\},
$$

$B(Y, \varepsilon)=\{x \in E: d(x, Y)<\varepsilon\}$, and $\inf \emptyset=\infty$. If $F: X \rightarrow E$ is a multi-valued map, then $x$ is called a fixed point of $F$ if $x \in F(x)$. The first fixed point result for multi-valued contraction was obtained by Nadler [43].

Theorem 3.1 (Nadler, 1969). Let $F: E \rightarrow E$ be a multi-valued contraction with closed bounded values, i.e., there exists $k<1$ such that

$$
D(F(x), F(y)) \leq k d(x, y) \quad \text { for every } x, y \in E .
$$

Then $F$ has a fixed point which can be obtained by iteration.

Proof. Fix $\varepsilon>0$ and $x_{0} \in E$. We define inductively a sequence $\left\{x_{n}\right\}$ satisfying

(a) ${ }_{n} x_{n} \in F\left(x_{n-1}\right)$;

(b) ${ }_{n} d\left(x_{n}, x_{n-1}\right)<k^{n-1}\left(d\left(x_{1}, x_{0}\right)+\varepsilon\right)$.

Choose $x_{1} \in F\left(x_{0}\right)$. Now, assume that there exist $x_{i}$ satisfying $(\mathrm{a})_{i},(\mathrm{~b})_{i}$ for $1 \leq i \leq n$. Since

$$
D\left(F\left(x_{n}\right), F\left(x_{n-1}\right)\right) \leq k d\left(x_{n}, x_{n-1}\right)<k^{n}\left(d\left(x_{1}, x_{0}\right)+\varepsilon\right),
$$

there exists $x_{n+1}$ satisfying $(\mathrm{a})_{n+1},(\mathrm{~b})_{n+1}$.

The inequality

$$
d\left(x_{n}, x_{n+p}\right)<\left(1+k+\ldots+k^{p-1}\right) k^{n}\left(d\left(x_{1}, x_{0}\right)+\varepsilon\right) \text { for all } n, p \in \mathbb{N}
$$

implies that $\left\{x_{n}\right\}$ is a Cauchy sequence, and hence converges to some $x \in E$. On the other hand, since $F(x)$ is closed and $D\left(F\left(x_{n}\right), F(x)\right) \leq k d\left(x_{n}, x\right)$, we deduce that $x \in F(x)$.

In the same paper, Nadler showed that the condition of contraction can be weakened if stronger assumptions are imposed on the space; more precisely if the space is $\varepsilon$-chainable (the definition can be found in Theorem 1.4(b)). 
THEOREM 3.2 (Nadler, 1969). Let $F: E \rightarrow E$ be a multi-valued mapping with closed bounded values such that there exist $\varepsilon>0$ and $k<1$ such that $F$ is $(\varepsilon, k)$-uniformly locally contractive, i.e.,

$$
d(x, y)<\varepsilon \Longrightarrow D(F(x), F(y)) \leq k d(x, y) .
$$

If $E$ is $\varepsilon$-chainable, then $F$ has a fixed point.

One year later, he showed with Covitz [12] that in the two previous results, the values of $F$ do not need to be bounded. Nadler's result was generalized by Reich in the spirit of Theorem 1.5 (see [53]), and also by replacing the condition of contraction as stated below (see [52]). If fact, the constants $k, l, m$ in the following theorem can be replaced by suitable functions of $d(x, y)$ when $E$ is compact (see [54]).

THEOREM 3.3 (Reich, 1971). Let $F: E \rightarrow E$ be a multi-valued mapping with closed values such that there exist $k, l, m \geq 0$ with $k+l+m<1$ such that for every $x, y \in E$,

$$
D(F(x), F(y)) \leq k d(x, y)+l \operatorname{dist}(x, F(x))+m \operatorname{dist}(y, F(y)) .
$$

Then $F$ has a fixed point.

This result was recently generalized by Rus, Petruşel and Sîntămărian [61], by replacing the condition by:

there exist $k, l, m \geq 0$ with $k+l+m<1$ such that for every $x \in E, u_{x} \in F(x)$, and every $y \in E$, there exists $u_{y} \in F(y)$ such that

$$
d\left(u_{x}, u_{y}\right) \leq k d(x, y)+l d\left(x, u_{x}\right)+m d\left(y, u_{y}\right) .
$$

Edelstein's periodic point result [16] was recently extended to multi-valued maps by Nadler [44]. He also obtained a fixed point result for compact and connected spaces.

THEOREM 3.4 (Nadler, 2003). Let $E$ be a metric space and $F: E \rightarrow E$ with compact values such that there exists $\varepsilon>0$ with

$$
d(x, y)<\varepsilon \Longrightarrow D(F(x), F(y))<d(x, y) \quad \text { for every } x \neq y .
$$

(a) If $E$ is compact and connected, then $F$ has a fixed point.

(b) Assume there exists $X \subset E$ compact such that $\left\{F^{n}(X)\right\}$ has a subsequence converging to some compact set $Y$. Then there exists $x_{0} \in Y$, a periodic point of $F$, i.e., there exist $m \in \mathbb{N}$ and $x_{1}, \ldots, x_{m}$ such that $x_{0} \in F\left(x_{m}\right)$ and $x_{i} \in F\left(x_{i-1}\right), i=1, \ldots, m$.

It is worthwhile to notice that Nadler showed that statement (b) is false if the compactness of the values of $F$ is replaced by closed bounded values.

3.2. Maps defined on subsets of $E$. Now, we consider multi-valued contractions defined on a subset of the space $E$. In order to guarantee the existence of a fixed point, extra assumptions will be needed. We state a result of Assad and Kirk [3] obtained in metrically convex complete metric space.

THEOREM 3.5 (Assad and Kirk, 1972). Let E be a metrically convex complete metric space, $X \subset E$ and $F: X \rightarrow E$ a contraction with closed bounded values. Assume that $F(x) \subset X$ for every $x \in \partial X$, then $F$ has a fixed point. 
This result was generalized by Matkowski [40] for maps $F$ such that there exists $\psi:[0, \infty) \rightarrow[0, \infty)$, continuous at 0 , such that there exist $k<1$ and $t_{n} \rightarrow 0$ with

$$
\psi\left(t_{n}\right) \leq k t_{n} \quad \text { and } \quad D(F(x), F(y)) \leq \psi(d(x, y)) \quad \forall x, y \in X .
$$

With no restriction on the space, Reich [56] obtained the following result for weakly inward maps. Let us mention that Caristi [10] gave an elementary proof of the following result for single-valued contractions.

Theorem 3.6 (Reich, 1978). Let $E$ be a Banach space, $X \subset E$ closed convex, and $F: X \rightarrow E$ a contraction with compact values which is weakly inward, i.e.,

$$
F(x) \subset \overline{I_{X}(x)}=\operatorname{cl}\{y \in E: y=x+t(z-x) \text { for some } z \in X, t \geq 0\} .
$$

Then $F$ has a fixed point.

Proof. Suppose $F$ has no fixed point. Choose $r \in(0,1)$ such that the constant of contraction satisfies $k<(1-r) /(1+r)$. For $x \in X$, there exists $z \in F(x)$ such that $\operatorname{dist}(x, F(x))=\|x-z\|$. Since $z \in \overline{I_{X}(x)}$, there exists $t \in(0,1)$ such that $w=t z+(1-t) x$ satisfies $\operatorname{dist}(w, X)<r t\|x-z\|$, see [10]. So, there exists $y \in X$ such that

$$
\|w-y\|<r t\|x-z\|=r\|w-x\| \quad \text { and } \quad\|x-y\|<(1+r)\|w-x\| .
$$

Moreover

$$
\begin{aligned}
\operatorname{dist}(y, F(y)) & \leq\|y-w\|+\operatorname{dist}(w, F(x))+D(F(x), F(y)) \\
& \leq\|y-w\|+\|w-z\|+k\|x-y\| \\
& \leq\|y-w\|+(1-t)\|x-z\|+k\|x-y\| \\
& <r\|w-x\|+\|x-z\|-\|w-x\|+k\|x-y\| \\
& =\|x-z\|-(1-r)\|w-x\|+k\|x-y\| \\
& <\operatorname{dist}(x, F(x))-c\|x-y\|,
\end{aligned}
$$

with $c=-k+(1-r) /(1+r)$. This defines a map $g: X \rightarrow X$ by $g(x)=y$ satisfying

$$
\|x-g(x)\|<\frac{1}{c}(\operatorname{dist}(x, F(x))-\operatorname{dist}(g(x), F(g(x)))) .
$$

The lower semi-continuity of the map $u \mapsto \operatorname{dist}(u, F(u)) / c$ and Caristi's fixed point theorem (see [32] for a simple proof) imply that $g$ has a fixed point; this contradicts (3.1).

It is natural to ask if this result is true when the values of $F$ are not compact but only closed and bounded. To my knowledge, the answer is still unknown. However, a partial answer was obtained by Mizoguchi and Takahashi [41] using a characterization of single-valued inward map, see [55] or [10]. Indeed, a single-valued map $f: X \rightarrow E$ is weakly inward (i.e., $f(x) \subset \overline{I_{X}(x)}$ for all $x \in X$ ) if and only if

$$
\lim _{t \rightarrow 0^{+}} \frac{\operatorname{dist}((1-t) x+t f(x), X)}{t}=0 \quad \text { for every } x \in X .
$$


Theorem 3.7 (Mizoguchi and Takahashi, 1989). Let $E$ be a Banach space, $X \subset E$ closed convex, and $F: X \rightarrow E$ a contraction with closed bounded values such that for every $x \in X$,

$$
\lim _{t \rightarrow 0^{+}} \frac{\operatorname{dist}((1-t) x+t y, X)}{t}=0 \quad \text { uniformly for } y \in F(x) .
$$

Then $F$ has a fixed point.

3.3. Stability results. Similarly to what was done for single-valued contractions, stability results for multi-valued contractions can be obtained. The first result in this direction was obtained by Nadler [43].

TheOREm 3.8 (Nadler, 1969). For $n \in \mathbb{N} \cup\{0\}$, let $F_{n}: E \rightarrow E$ be a multi-valued contraction with closed bounded values with constant of contraction $k_{n}<1$. Assume that one of the following statements holds:

(a) $F_{n} \rightarrow F_{0}$ uniformly;

(b) $\sup k_{n}<1$ and $F_{n} \rightarrow F_{0}$ pointwise;

(c) $E$ is locally compact and $F_{n} \rightarrow F_{0}$ pointwise.

Then there exists a sequence $\left\{x_{n_{m}}\right\}$ such that $x_{n_{m}} \in F_{n_{m}}\left(x_{n_{m}}\right)$ and $x_{n_{m}} \rightarrow x_{0} \in F_{0}\left(x_{0}\right)$.

With stronger assumptions, more precision on the behavior of $\operatorname{Fix}\left(F_{n}\right)$ was obtained by $\operatorname{Lim}[39]$.

Theorem 3.9 (Lim, 1985). For $n \in \mathbb{N} \cup\{0\}$, let $F_{n}: E \rightarrow E$ be a multi-valued contraction with closed bounded values with constant of contraction $k_{n}<1$ such that $\sup k_{n}<1$ and $F_{n} \rightarrow F_{0}$ uniformly. Then

$$
\lim _{n \rightarrow \infty} D\left(\operatorname{Fix}\left(F_{n}\right), \operatorname{Fix}\left(F_{0}\right)\right)=0 .
$$

Proof. Let $k=\sup k_{n}<1$. For $n \in \mathbb{N} \cup\{0\}$, from the proof of Theorem 3.1, we deduce that for every $x \in E$ and every $y \in F_{n}(x)$, there exists $z \in \operatorname{Fix}\left(F_{n}\right)$ such that

$$
d(x, z) \leq \frac{d(x, y)}{1-k} .
$$

In particular, for $m \in \mathbb{N}, z \in \operatorname{Fix}\left(F_{n}\right)$ and for every $y \in F_{m}(z)$, there exists $z_{y} \in \operatorname{Fix}\left(F_{m}\right)$ such that

$$
d\left(z, z_{y}\right) \leq \frac{d(z, y)}{1-k} .
$$

For $\varepsilon>0$, there exists $\hat{y} \in F_{m}(z)$ such that

$$
d(z, \hat{y}) \leq D\left(F_{n}(z), F_{m}(z)\right)+\varepsilon .
$$

So, denoting $\hat{z}=z_{\hat{y}} \in \operatorname{Fix}\left(F_{m}\right)$, we have that for every $\varepsilon>0$,

$$
\forall z \in \operatorname{Fix}\left(F_{n}\right), \exists \hat{z} \in \operatorname{Fix}\left(F_{m}\right) \text { such that } \quad d(z, \hat{z}) \leq \frac{D\left(F_{n}(z), F_{m}(z)\right)+\varepsilon}{1-k} .
$$

Since $\varepsilon$ is arbitrary and interchanging $n$ and $m$ permits us to deduce that for every $m \in \mathbb{N}, n \in \mathbb{N} \cup\{0\}$,

$$
D\left(\operatorname{Fix}\left(F_{n}\right), \operatorname{Fix}\left(F_{m}\right)\right) \leq(1-k)^{-1} \sup _{x \in E} D\left(F_{n}(x), F_{m}(x)\right) .
$$


Rus, Petruşel and Sîntămărian [61] extended Lim's result by introducing a generalization of contractive mappings.

Definition 3.10. Let $F: E \rightarrow E$ be a multi-valued mapping and $k \geq 0$. The map $F$ is called a $k$-multi-valued weakly Picard operator $(k-M W P)$ if for every $x_{0} \in E$ and $x_{1} \in F\left(x_{0}\right)$, there exists

$$
f^{\infty}\left(x_{0}, x_{1}\right) \in\left\{y \in E: \exists x_{n+1} \in F\left(x_{n}\right), \text { for } n \geq 1 \text { with } x_{n} \rightarrow y \in F(y)\right\}
$$

such that

$$
d\left(x_{0}, f^{\infty}\left(x_{0}, x_{1}\right)\right) \leq k d\left(x_{0}, x_{1}\right) .
$$

In particular, if $E$ is complete and $F$ has closed values and satisfies Reich's condition: there exist $k, l, m \geq 0$ with $k+l+m<1$ such that

$$
D(F(x), F(y)) \leq k d(x, y)+l \operatorname{dist}(x, F(x))+m \operatorname{dist}(y, F(y)),
$$

then $F$ is $c$-MWP with $c=\frac{1-m}{1-k-l-m}$.

Theorem 3.11 (Rus, Petruşel and Sîntămărian, 2003). Let E be a metric space (not necessarily complete), $F_{i}: E \rightarrow E$ a $k_{i}-M W P$, for $i=1,2$. Assume that there exists $\delta>0$ such that $D\left(F_{1}(x), F_{2}(x)\right) \leq \delta$ for all $x \in E$. Then

$$
D\left(\operatorname{Fix}\left(F_{1}\right), \operatorname{Fix}\left(F_{2}\right)\right) \leq \delta \max \left\{k_{1}, k_{2}\right\} .
$$

4. Continuation results for multi-valued contractions. In this section, we present a continuation result for multi-valued contractions obtained in [22]. Let us mention that this result was known in Banach spaces for $k$-set contractions with a non-elementary proof.

Theorem 4.1 (Frigon and Granas, 1994). Let $E$ be a complete metric space, $U \subset E$ open, and $H: \bar{U} \times[0,1] \rightarrow E$ such that

(i) there exists $k<1$ such that $D(H(x, t), H(y, t)) \leq k d(x, y)$ for every $x, y \in E$ and $t \in[0,1]$;

(ii) there exists $\phi:[0,1] \rightarrow \mathbb{R}$ continuous and increasing such that

$$
D(H(x, t), H(x, s)) \leq|\phi(t)-\phi(s)| \quad \text { for every } x \in E \text { and } s, t \in[0,1] ;
$$

(iii) $x \notin H(x, t)$ for all $(x, t) \in \partial U \times[0,1]$.

If $H(\cdot, 0)$ has a fixed point then $H(\cdot, t)$ has a fixed point for each $t \in[0,1]$.

Proof. Let us consider the set

$$
Q=\{(t, x) \in[0,1] \times U: x \in H(x, t)\} .
$$

By assumption $(0, \bar{x}) \in Q$ where $\bar{x} \in H(\bar{x}, 0)$.

On $Q$ we define the partial order:

$$
(t, x) \leq(s, y) \quad \text { if and only if } t \leq s \text { and } d(x, y) \leq \frac{2(\phi(s)-\phi(t))}{1-k} .
$$


Let $P$ be a totally ordered subset of $Q$. Define $t^{*}=\sup \{t:(t, x) \in P\}$. Take a sequence $\left\{\left(t_{n}, x_{n}\right)\right\}$ in $P$ such that $\left(t_{n}, x_{n}\right) \leq\left(t_{n+1}, x_{n+1}\right)$ and $t_{n} \rightarrow t^{*}$. We have

$$
d\left(x_{m}, x_{n}\right) \leq \frac{2\left(\phi\left(t_{m}\right)-\phi\left(t_{n}\right)\right)}{1-k} \quad \text { for all } m>n .
$$

Thus, $\left\{x_{n}\right\}$ is a Cauchy sequence, and hence converges to some $x^{*} \in \bar{U}$. The continuity of $H$ implies that $\left(t^{*}, x^{*}\right) \in Q$. Moreover, it is clear that

$$
(t, x) \leq\left(t^{*}, x^{*}\right) \quad \text { for every }(t, x) \in P .
$$

That means that $\left(t^{*}, x^{*}\right)$ is an upper bound of $P$. It follows from Zorn's Lemma that $Q$ admits a maximal element $\left(t_{0}, x_{0}\right) \in Q$.

To complete the proof, we have to show that $t_{0}=1$. Suppose this is false. Then, we can choose $r>0$ and $t \in\left(t_{0}, 1\right]$ such that

$$
\overline{B\left(x_{0}, r\right)} \subset U, \quad \text { and } \quad r>\frac{\left(\phi(t)-\phi\left(t_{0}\right)\right)}{1-k} .
$$

It follows that

$$
\begin{array}{r}
\operatorname{dist}\left(x_{0}, H\left(x_{0}, t\right)\right) \leq \operatorname{dist}\left(x_{0}, H\left(x_{0}, t_{0}\right)\right)+D\left(H\left(x_{0}, t_{0}\right), H\left(x_{0}, t\right)\right) \\
\quad \leq \phi(t)-\phi\left(t_{0}\right)<(1-k) r .
\end{array}
$$

Arguing as in the proof of Theorem 3.1, we define inductively a sequence in $\overline{B\left(x_{0}, r\right)}$ converging to $x$ a fixed point of $H(\cdot, t)$. Thus, $(t, x) \in Q$ and $\left(t_{0}, x_{0}\right)<(t, x)$; this contradicts the maximality of $\left(t_{0}, x_{0}\right)$.

As before, the contraction condition can be generalized for example by the condition

$\exists k<1$ such that $D(H(x, t), H(y, t)) \leq k \max \{d(x, y), \operatorname{dist}(x, H(x, t))$,

$$
\left.\operatorname{dist}(y, H(y, t)), \frac{1}{2}[\operatorname{dist}(x, H(y, t))+\operatorname{dist}(y, H(x, t))]\right\} .
$$

This condition was used by O'Regan and myself [25] to generalize the previous result. This permits us to apply our result to fuzzy contractive maps. Condition (ii) on the continuity with respect to the parameter can also be weakened if a boundary condition stronger than (iii) is imposed, see Agarwal and O'Regan [2] for more details.

5. Single-valued contractions in gauge spaces. We are interested to study contractions in more general spaces, and in particular in a gauge space, i.e., a topological space with a gauge structure, that is such that its topology is induced by $\left\{d_{\alpha}\right\}_{\alpha \in \Lambda}$, a separating family of gauges (also called semi-metric, pseudometric, ecart, see [13]).

We start with a result of Cain and Nashed [9] in Hausdorff locally convex topological spaces.

TheOREM 5.1 (Cain and Nashed, 1971). Let $\left(E,\left\{\|\cdot\|_{\alpha}\right\}_{\alpha \in \Lambda}\right)$ be a Hausdorff locally convex topological vector space, $X \subset E$ sequentially complete, and $f: X \rightarrow X$ a contraction, i.e.,

$$
\forall \alpha \in \Lambda \exists k_{\alpha}<1 \text { such that }\|f(x)-f(y)\|_{\alpha} \leq k_{\alpha}\|x-y\|_{\alpha} \quad \forall x, y \in X .
$$

Then $f$ has a unique fixed point. 
Proof. For every $y \in X$,

$$
\left\|f^{n}(y)-y\right\|_{\alpha} \leq \frac{\|f(y)-y\|_{\alpha}}{1-k_{\alpha}} \quad \text { for every } n \in \mathbb{N} \text { and } \alpha \in \Lambda .
$$

Hence, for $x \in X, y=f^{m}(x)$ and $m \in \mathbb{N}$,

$$
\left\|f^{m+n}(x)-f^{m}(x)\right\|_{\alpha} \leq \frac{\left\|f^{m+1}(x)-f^{m}(x)\right\|_{\alpha}}{1-k_{\alpha}} \leq \frac{k^{m}\|f(x)-x\|_{\alpha}}{1-k_{\alpha}} \quad \forall n \in \mathbb{N}, \forall \alpha \in \Lambda .
$$

Thus, $\left\{f^{n}(x)\right\}$ is a Cauchy sequence in $X$ and hence converges to some $\hat{x} \in X$. Clearly, $\hat{x}$ is the unique fixed point of $f$.

Observe that in the particular case where $\Lambda$ is countable then $\left(E,\left\{\|\cdot\|_{\alpha}\right\}_{\alpha \in \Lambda}\right)$ is metrizable. However, $f$ can be a contraction in the sense of the Theorem 5.1 without being a contraction with the metric induced by the topology of $E$.

Let us mention that this result was not the first one of this type. Indeed, in 1965, Knill [38] gave a notion of contraction and a fixed point result in Hausdorff uniform spaces.

In 1974, Tarafdar [62] expressed the notion of contraction in Hausdorff uniform spaces as follows:

$$
\forall \alpha \in \Lambda \exists k_{\alpha}<1 \text { such that } d_{\alpha}(f(x), f(y)) \leq k_{\alpha} d_{\alpha}(x, y) \forall x, y \in E,
$$

using the observation that a uniformity on $E$ determines a family of gauges or pseudometrics $\left\{d_{\alpha}\right\}_{\alpha \in \Lambda}$. So, the Banach contraction principle can be extended to complete Hausdorff uniform spaces or complete gauge spaces $\left(E,\left\{d_{\alpha}\right\}_{\alpha \in \Lambda}\right)$.

Continuation results in gauge spaces for $h: \bar{U} \times[0,1] \rightarrow E$ can also be obtained as in Theorem 2.3 when $h(\cdot, t)$ are contractions with the same constants $\left\{k_{\alpha}\right\}$ and $U$ is open in $E$, see [23]. To my knowledge, there are no applications of this result in this generality. The problem is that in gauge spaces, open sets are too big. Indeed, in eventual applications, we have to consider maps $f$ defined on a closed subset $X$ of the gauge space $E$ which has empty interior. A result for weakly inward map defined on a closed subset of a Fréchet space and satisfying an appropriate differential equation was obtained by Reich [57], see [55] for a similar result in Banach spaces for single-valued contractions.

Theorem 5.2 (Reich, 1980). Let $\left(E,\left\{\|\cdot\|_{n}\right\}_{n \in \mathbb{N}}\right)$ be a Fréchet space, $X \subset E$ bounded, closed, convex, and $f: X \rightarrow E$ a k-set contraction which is weakly inward, i.e., $f(x) \in \overline{I_{X}(x)}$ for all $x \in X$. Assume for every $C$ closed and convex such that $\left.f\right|_{C}$ is weakly inward, the initial value problem $u^{\prime}(t)=f(u(t))-u(t), u(0)=x_{0}$ has a $C^{1}$-solution $u:[0, \infty) \rightarrow C$ for every $x_{0} \in C$. Then $f$ has a fixed point.

Idea of the proof. Construct $K=C_{1} \supset C_{2} \supset \ldots$ such that $C_{i}$ is closed convex, the measure of compactness $\alpha\left(C_{i}\right)<1 / i$, and $\left.f\right|_{C_{i}}$ is weakly inward. Take $C=\bigcap C_{i}$, hence $C$ is compact convex. Using the differential equation and Ascoli's theorem, show that $\left.f\right|_{C}$ is weakly inward and apply Halpern-Bergman's theorem [33].

Polewczak [47] showed that, in the previous result, the condition on the differential equation is not necessary if $f$ is a contraction satisfying a stronger condition than weakly inwardness. 
TheOrem 5.3 (Polewczak, 1989). Let $\left(E,\left\{\|\cdot\|_{n}\right\}_{n \in \mathbb{N}}\right)$ be a Fréchet space, $X \subset E$ closed and locally bounded, and $f: X \rightarrow E$ a contraction such that

$$
\lim _{h \rightarrow 0^{+}} \frac{\operatorname{dist}((1-h) x+h f(x), X)}{h}=0 \quad \forall x \in X,
$$

where $\operatorname{dist}(z, X)=\inf \{\|x-z\|: x \in X\}$, and $\|z\|=\sum_{n=1}^{\infty} \frac{c_{n}\|z\|_{n}}{1+\|z\|_{n}}$ for a fixed positive sequence $\left\{c_{n}\right\}$ such that $\sum_{n=1}^{\infty} c_{n}<\infty$. Then $f$ has a unique fixed point.

It appears that the notion of contraction in gauge spaces is very restrictive. Indeed, if

$$
\begin{aligned}
\left(E,\left\{\|\cdot\|_{n}\right\}\right)= & \left(\prod_{n \in \mathbb{N}} B_{n},\left\{\|\cdot\|_{n}\right\}\right) \text { with }\left(B_{n},|\cdot|_{n}\right) \text { Banach spaces, } \\
& \left\|\left(x_{1}, x_{2}, \ldots\right)\right\|_{n}=\left|x_{1}\right|_{1}+\cdots+\left|x_{n}\right|_{n},
\end{aligned}
$$

and $f=\left(f_{1}, f_{2}, \ldots\right): E \rightarrow E$ a contraction then $f_{n}$ does not depend on $x_{n+1}, x_{n+2}, \ldots$ In particular, if $f$ is a contraction such that

$$
\left(\begin{array}{c}
\left|f_{1}(x)-f_{1}(y)\right|_{1} \\
\left|f_{2}(x)-f_{2}(y)\right|_{2} \\
\vdots
\end{array}\right) \leq A\left(\begin{array}{c}
\left|x_{1}-y_{1}\right|_{1} \\
\left|x_{2}-y_{2}\right|_{2} \\
\vdots
\end{array}\right)
$$

where $A=\left(a_{i, j}\right)$ is a matrix such that $a_{i, j} \geq 0$, then $A$ is lower triangular.

In the following result, Gheorghiu [28] permits row finite matrix $A$. He considers also two gauge structures on $E$.

THEOREM 5.4 (Gheorghiu, 1982). Let $E$ be a set endowed with two gauge structures $\mathcal{D}_{0}=\left\{\hat{d}_{\alpha}\right\}_{\alpha \in \Lambda_{0}}, \mathcal{D}_{1}=\left\{d_{\alpha}\right\}_{\alpha \in \Lambda_{1}}$, and $f: E \rightarrow E$ such that

(i) $\left(E, \mathcal{D}_{0}\right)$ is sequentially complete, $f:\left(E, \mathcal{D}_{0}\right) \rightarrow\left(E, \mathcal{D}_{1}\right)$ is sequentially continuous;

(ii) there exists $\phi: \Lambda_{1} \rightarrow \Lambda_{1}$, and for all $\alpha \in \Lambda_{1}$, there exists $k_{\alpha}<1$ such that

$$
d_{\alpha}(f(x), f(y)) \leq k_{\alpha} d_{\phi(\alpha)}(x, y) \quad \text { for every } x, y \in E,
$$

and

$$
\sum_{n=1}^{\infty} k_{\alpha} k_{\phi(\alpha)} \ldots k_{\phi^{n-1}(\alpha)} d_{\phi^{n}(\alpha)}(x, y)<\infty ;
$$

(iii) there exists $\psi: \Lambda_{0} \rightarrow \Lambda_{1}$ and for all $\alpha \in \Lambda_{0}$, there exists $c_{\alpha}$ such that

$$
\hat{d}_{\alpha}(x, y) \leq c_{\alpha} d_{\psi(\alpha)}(x, y) \quad \text { for every } x, y \in E .
$$

Then $f$ has a unique fixed point which can be obtained by iteration.

Chiş and Precup [11] obtained a continuation result for that type of map.

Again, we can raise the question if a more general matrix $A$ can be considered and in particular with all $a_{i, j}>0$. To this aim, we recall the notion of generalized contraction introduced in [20].

We consider $\left(E,\left\{d_{\alpha}\right\}_{\alpha \in \Lambda}\right)$ a complete gauge space satisfying

$$
\Lambda \text { is a directed set, such that } d_{\alpha}(x, y) \leq d_{\beta}(x, y) \text { if } \alpha \leq \beta \text {. }
$$


In fact, $E$ is the projective limit of a family of Banach spaces $\left\{E_{\alpha}\right\}_{\alpha \in \Lambda}$ where $E_{\alpha}$ is the completion of $E / \sim_{\alpha}$ with respect to $d_{\alpha}$ and for the equivalence relation $\sim_{\alpha}$ defined by

$$
x \sim_{\alpha} y \quad \Longleftrightarrow \quad d_{\alpha}(x, y)=0 .
$$

This defines a continuous map $[\cdot]_{\alpha}: E \rightarrow E_{\alpha}$. Similarly, for every $\beta \geq \alpha \in \Lambda$, we define $\operatorname{maps}[\cdot]_{\alpha \beta}: E_{\beta} \rightarrow E_{\alpha}$.

For $X \subset E$, we set $X_{\alpha}=\left\{[x]_{\alpha}: x \in X\right\}$, we denote by $\overline{X_{\alpha}}$, and $\partial X_{\alpha}$, respectively, the closure, and the boundary of $X_{\alpha}$ with respect to $d_{\alpha}$ in $E_{\alpha}$. For $x \in X$, we set $\{x\}_{\alpha}=\left\{y \in X: d_{\alpha}(x, y)=0\right\}$.

We define a family of generalized gauges on the space of subsets of $E$ by

$$
D_{\alpha}(X, Y)=\inf \left\{\varepsilon>0: \varepsilon \in \mathcal{E}_{\alpha}(X, Y)\right\},
$$

where

$$
\mathcal{E}_{\alpha}(X, Y)=\left\{\varepsilon>0: X \subset B_{\alpha}(Y, \varepsilon), Y \subset B_{\alpha}(X, \varepsilon)\right\}
$$

with

$$
B_{\alpha}(X, r)=\left\{y \in E: \operatorname{dist}_{\alpha}(y, X)<r\right\}
$$

and

$$
\operatorname{dist}_{\alpha}(y, X)=\inf \left\{d_{\alpha}(x, y): x \in X\right\} ;
$$

and $\inf \emptyset=\infty$. We define

$$
\operatorname{diam}_{\alpha}(X)=\inf \left\{d_{\alpha}(x, y): x, y \in X\right\} .
$$

Definition 5.5. A function $f: X \rightarrow E$ is a generalized contraction if

(i) for every $\alpha \in \Lambda$, there exists $k_{\alpha}<1$ such that

$$
D_{\alpha}\left(f\left(\{x\}_{\alpha}\right), f\left(\{y\}_{\alpha}\right)\right) \leq k_{\alpha} d_{\alpha}(x, y) \text { for every } x, y \in X
$$

(ii) for every $\varepsilon>0$ and every $\alpha \in \Lambda$, there exists $\beta \geq \alpha$ such that

$$
\operatorname{diam}_{\beta}\left(f\left(\{x\}_{\alpha}\right)\right)<\left(1-k_{\beta}\right) \varepsilon \quad \text { for every } x \in X \text {. }
$$

We will also say that $k=\left(k_{\alpha}\right)_{\alpha \in \Lambda} \in[0,1)^{\Lambda}$ is a constant of contraction of $f$.

Observe that if $f: X \rightarrow E$ is a generalized contraction then for every $\alpha \in \Lambda$, we can define a multi-valued map with closed values $F_{\alpha}: \overline{X_{\alpha}} \rightarrow E_{\alpha}$ which is the continuous extension of

$$
[x]_{\alpha} \mapsto \operatorname{cl}\left\{[f(y)]_{\alpha}: y \in\{x\}_{\alpha}\right\} \quad \text { for }[x]_{\alpha} \in X_{\alpha} .
$$

The map $F_{\alpha}$ is a multi-valued contraction with constant $k_{\alpha}$.

THEOREM 5.6 (Frigon, 2000). Let $\left(E,\left\{d_{\alpha}\right\}_{\alpha \in \Lambda}\right)$ be a complete gauge space satisfying (5.1), and $f: E \rightarrow E$ a generalized contraction. Then $f$ has a unique fixed point.

Proof. Since $f$ is a generalized contraction, for every $\alpha \in \Lambda$, the map $F_{\alpha}$ defined above is a multi-valued contraction in a complete metric space. By Nadler's fixed point theorem (Theorem 3.1), it has a fixed point $z_{\alpha} \in F_{\alpha}\left(z_{\alpha}\right)$. Obviously, $\left[z_{\beta}\right]_{\alpha \beta} \in F_{\alpha}\left(\left[z_{\beta}\right]_{\alpha \beta}\right)$ for all $\beta \geq \alpha$.

Let $\varepsilon>0$, and $\gamma \in \Lambda$. Since $f$ is a generalized contraction, there exists $\alpha \geq \gamma$ such that

$$
\operatorname{diam}_{\alpha}\left(F_{\alpha}(z)\right)<\left(1-k_{\alpha}\right) \varepsilon \text { for all } z \in E_{\alpha} \text {. }
$$


It follows that for $\lambda, \beta \geq \alpha$,

$$
\begin{aligned}
d_{\alpha}\left(\left[z_{\lambda}\right]_{\alpha \lambda},\left[z_{\beta}\right]_{\alpha \beta}\right) \leq D_{\alpha}\left(F_{\alpha}\left(\left[z_{\lambda}\right]_{\alpha \lambda}\right), F_{\alpha}\left(\left[z_{\beta}\right]_{\alpha \beta}\right)\right) & +\operatorname{diam}_{\alpha}\left(F_{\alpha}\left(\left[z_{\lambda}\right]_{\alpha \lambda}\right)\right) \\
& <k_{\alpha} d_{\alpha}\left(\left[z_{\lambda}\right]_{\alpha \lambda},\left[z_{\beta}\right]_{\alpha \beta}\right)+\left(1-k_{\alpha}\right) \varepsilon
\end{aligned}
$$

Hence,

$$
d_{\gamma}\left(\left[z_{\lambda}\right]_{\gamma \lambda},\left[z_{\beta}\right]_{\gamma \beta}\right) \leq d_{\alpha}\left(\left[z_{\lambda}\right]_{\alpha \lambda},\left[z_{\beta}\right]_{\alpha \beta}\right)<\varepsilon .
$$

So, for all $\gamma \in \Lambda,\left\{\left[z_{\alpha}\right]_{\gamma \alpha}\right\}_{\alpha \geq \gamma}$ is a Cauchy net in $E_{\gamma}$, and therefore, converges to some $x \in E$.

We claim that $x=f(x)$. Indeed, let $\gamma \in \Lambda$. For $\varepsilon>0$, choose $\beta \geq \alpha \geq \gamma$ such that $\operatorname{diam}_{\alpha}\left(f\left(\{x\}_{\alpha}\right)\right)<\varepsilon / 2$, and $d_{\alpha}\left([x]_{\alpha},\left[z_{\beta}\right]_{\alpha \beta}\right)<\varepsilon / 4$. We have

$$
\begin{aligned}
d_{\gamma}(x, f(x)) & \leq d_{\alpha}(x, f(x)) \\
& \leq d_{\alpha}\left([x]_{\alpha},\left[z_{\beta}\right]_{\alpha \beta}\right)+D_{\alpha}\left(F_{\alpha}\left(\left[z_{\beta}\right]_{\alpha \beta}\right), F_{\alpha}\left([x]_{\alpha}\right)\right)+\operatorname{diam}_{\alpha}\left(F_{\alpha}\left([x]_{\alpha}\right)\right) \\
& \leq\left(1+k_{\alpha}\right) d_{\alpha}\left([x]_{\alpha},\left[z_{\beta}\right]_{\alpha \beta}\right)+\frac{\varepsilon}{2} \leq \varepsilon .
\end{aligned}
$$

A similar argument permits to deduce the uniqueness of $x$, the fixed point of $f$.

Now, we present a continuation result on a subset $X$ which can have empty interior.

THEOREM 5.7 (Frigon, 2000). Let $\left(E,\left\{d_{\alpha}\right\}_{\alpha \in \Lambda}\right)$ be a complete gauge space satisfying (5.1), $X$ a closed subset of $E$, and $h: X \times[0,1] \rightarrow E$ such that

(i) there exists $k \in[0,1)^{\Lambda}$ such that $h(\cdot, t)$ is a generalized contraction with constant of contraction $k$, for every $t \in[0,1]$;

(ii) for every $\alpha \in \Lambda$, there exists $M_{\alpha} \geq 0$ such that

$$
D_{\alpha}\left(h\left(\{x\}_{\alpha}, t\right), h\left(\{x\}_{\alpha}, s\right)\right) \leq M_{\alpha}|t-s| \quad \text { for every } x \in X, s, t \in[0,1] ;
$$

(iii) $z \notin H_{\alpha}(z, t)$ for every $z \in \partial X_{\alpha}, t \in[0,1]$, and $\alpha \in \Lambda$, where $H_{\alpha}: \overline{X_{\alpha}} \times[0,1] \rightarrow E_{\alpha}$ is the multi-valued map obtained as above.

If $h(\cdot, 0)$ has a fixed point, then $h(\cdot, t)$ has a unique fixed point for all $t \in[0,1]$.

Proof. If $h(\cdot, 0)$ has a fixed point, then $H_{\alpha}(\cdot, 0)$ also has a fixed point for all $\alpha \in \Lambda$. The continuation principle for multi-valued contractions in Banach spaces (Theorem 4.1) implies that for every $t \in[0,1]$ and every $\alpha \in \Lambda, H_{\alpha}(\cdot, t)$ has a fixed point $x_{\alpha}^{t} \in E_{\alpha}$. By arguing as in the previous theorem, we deduce that the net $\left\{x_{\alpha}^{t}\right\}_{\alpha \in \Lambda}$ converges to some $x^{t} \in X$ such that $x^{t}=h\left(x^{t}, t\right)$.

REMARK 5.8. In the previous theorem, it is suitable to impose condition (iii) instead of (iii) $)^{\prime} x \neq h(x, t)$ for every $x \in \partial X, t \in[0,1]$.

Indeed, in many applications, $X$ has empty interior: in this case, (iii) ${ }^{\prime}$ means that $h(\cdot, t)$ has no fixed point for every $t$.

In Theorem 5.6, the fixed point of the generalized contraction $f$ was not obtained by iteration. I raised the question to know if it is possible to obtain the fixed point of $f$ as the limit of a sequence defined by iteration. Recently, Espínola and Kirk [17] gave a positive answer to this question and they generalized Theorem 5.6. 
Theorem 5.9 (Espínola and Kirk, 2003). Let $\left(E,\left\{d_{\alpha}\right\}_{\alpha \in \Lambda}\right)$ be a complete gauge space satisfying (5.1), and for every $\alpha \in \Lambda$, let $F_{\alpha}: E \rightarrow E$ be a multi-valued map with closed values. Assume that

(i) for every $\alpha \in \Lambda$, there exists $k_{\alpha}<1$ such that

$$
D_{\alpha}\left(F_{\alpha}(x), F_{\alpha}(y)\right) \leq k_{\alpha} d_{\alpha}(x, y) \quad \text { for every } x, y \in E ;
$$

(ii) $\beta \geq \alpha$ implies that $F_{\beta}(x) \subset F_{\alpha}(x)$ for every $x \in E$;

(iii) for every $\varepsilon>0$ and every $\alpha \in \Lambda$, there exists $\beta \geq \alpha$ such that

$$
\operatorname{diam}_{\beta}\left(F_{\beta}(x)\right) \leq\left(1-k_{\beta}\right) \varepsilon \text { for every } x \in E .
$$

Then there exists a unique $x_{0} \in E$ such that $x_{0} \in F_{\alpha}\left(x_{0}\right)$ for every $\alpha \in \Lambda$. Moreover $x \mapsto f(x)=\bigcap_{\alpha} F_{\alpha}(x)$ is a single-valued map, and $f^{n}(x) \rightarrow x_{0}$ for every $x \in E$.

Proof. Let $x \in E$. Observe that $f(x)$ is non-empty. Indeed, choose for $\alpha \in \Lambda, x_{\alpha} \in F_{\alpha}(x)$. Let $\varepsilon>0$ and $\alpha \in \Lambda$, condition (iii) implies that there exists $\beta \geq \alpha$ such that

$$
\operatorname{diam}_{\beta}\left(F_{\beta}(x)\right) \leq \varepsilon .
$$

So, for $\mu, \nu \geq \beta, x_{\mu}, x_{\nu} \in F_{\beta}(x)$ and hence $d_{\alpha}\left(x_{\mu}, x_{\nu}\right) \leq \varepsilon$. So $\left\{x_{\alpha}\right\}$ converges to some $y \in f(x)$. Again, condition (iii) implies that $f$ is single-valued.

Observe that for $\alpha \in \Lambda$ and $S \subset E$ with $\operatorname{diam}_{\alpha}(S)>0$, using condition (iii), we can choose $\beta \geq \alpha$ such that

$$
\operatorname{diam}_{\beta}\left(F_{\beta}(y)\right) \leq \frac{\left(1-k_{\beta}\right)}{4} \operatorname{diam}_{\alpha}(S) \text { for every } y \in E .
$$

So, for $u, v \in S$,

$$
\begin{aligned}
d_{\beta}(f(u), f(v)) & \leq D_{\beta}\left(F_{\beta}(u), F_{\beta}(v)\right)+\operatorname{diam}_{\beta}\left(F_{\beta}(u)\right)+\operatorname{diam}_{\beta}\left(F_{\beta}(v)\right) \\
& \leq k_{\beta} d_{\beta}(u, v)+\frac{\left(1-k_{\beta}\right)}{2} \operatorname{diam}_{\alpha}(S) \\
& \leq k_{\beta} \operatorname{diam}_{\beta}(S)+\frac{\left(1-k_{\beta}\right)}{2} \operatorname{diam}_{\alpha}(S) \\
& \leq \frac{\left(1+k_{\beta}\right)}{2} \operatorname{diam}_{\beta}(S) .
\end{aligned}
$$

Hence

$$
\operatorname{diam}_{\alpha}(f(S)) \leq \operatorname{diam}_{\beta}(f(S)) \leq \frac{\left(1+k_{\beta}\right)}{2} \operatorname{diam}_{\beta}(S) .
$$

On the other hand, observe that for $A$ and $F_{\beta}(A)$ bounded sets in $E$,

$$
D_{\beta}\left(F_{\beta}^{2}(A), F_{\beta}(A)\right) \leq k_{\beta} D_{\beta}\left(A, F_{\alpha}(A)\right) .
$$

Indeed, for $\varepsilon>0$ and for $y \in F_{\beta}(A)$, there exists $z \in A$ such that

$$
d_{\beta}(y, z) \leq D_{\beta}\left(A, F_{\beta}(A)\right)+\varepsilon .
$$

So,

$$
D_{\beta}\left(F_{\beta}(y), F_{\beta}(z)\right) \leq k_{\beta}\left(D_{\beta}\left(A, F_{\beta}(A)\right)+\varepsilon\right),
$$

Interchanging the roles of $y$ and $z$ and using the fact that $\varepsilon$ is arbitrary, we obtain that

$$
D_{\beta}\left(F_{\beta}^{2}(A), F_{\beta}(A)\right) \leq k_{\beta} D_{\beta}\left(A, F_{\beta}(A)\right) .
$$


Therefore $F_{\beta}^{2}(A)$ is bounded. Repeating the argument permits to conclude that

$$
D_{\beta}\left(F_{\beta}^{n+1}(A), F_{\beta}(A)\right) \leq \frac{1}{1-k_{\beta}} D_{\beta}\left(A, F_{\beta}(A)\right)=: \frac{r_{\beta}}{2} \quad \forall n \in \mathbb{N} .
$$

We conclude that

$$
F_{\beta}^{n}(A) \subset \overline{B_{\beta}\left(A, r_{\beta}\right)}=\left\{y \in E: \exists x \in A \text { such that } d_{\beta}(x, y) \leq r_{\beta}\right\} \quad \forall n \in \mathbb{N} .
$$

Let $x \in E$. For $n \in \mathbb{N}$, define

$$
C_{n}=\left\{f^{n}(x), f^{n+1}(x), \ldots\right\} .
$$

For $\alpha \in \Lambda, f^{n+1}(x) \in F_{\alpha}\left(f^{n}(x)\right) \subset F_{\alpha}^{n}(x)$ for every $n \in \mathbb{N}$ since $f(y) \in F_{\alpha}(y)$ for every $y \in E$. So, $C_{n+1} \subset F_{\alpha}\left(C_{n}\right)$ for every $n \in \mathbb{N}$ and $\alpha \in \Lambda$.

Let $\beta \geq \alpha$ be given by condition (iii) such that $F_{\beta}(x)$ is bounded and

$$
\operatorname{diam}_{\beta}\left(F_{\beta}(y)\right) \leq \frac{\left(1-k_{\beta}\right)}{4} \operatorname{diam}_{\alpha}\left(C_{n}\right) \text { for every } y \in E .
$$

It follows from (5.3) with $A=\{x\}$ that the orbit

$$
C_{1} \subset \overline{B_{\beta}\left(x, r_{\beta}\right)} \subset \overline{B_{\alpha}\left(x, r_{\beta}\right)} .
$$

Also, equation (5.2) implies that

$$
\operatorname{diam}_{\beta}\left(C_{n+1}\right) \leq \frac{\left(1+k_{\beta}\right)}{2} \operatorname{diam}_{\beta}\left(C_{n}\right) \text { for every } n \in \mathbb{N},
$$

and hence by (5.4), for all $n \in \mathbb{N}$,

$$
\operatorname{diam}_{\alpha}\left(C_{n+1}\right) \leq \operatorname{diam}_{\beta}\left(C_{n+1}\right) \leq\left(\frac{1+k_{\beta}}{2}\right)^{n} \operatorname{diam}_{\beta}\left(C_{1}\right) \leq 2 r_{\beta}\left(\frac{1+k_{\beta}}{2}\right)^{n} .
$$

This permits to conclude that $\left\{f^{n}(x)\right\}$ is a Cauchy sequence converging to some $z \in E$. Therefore, $z=f(z) \subset F_{\alpha}(z)$ for all $\alpha \in \Lambda$. If $y=f(y),(5.2)$ with $S=\{y, z\}$ implies that

$$
d_{\alpha}(y, z)=d_{\alpha}(f(y), f(z)) \leq d_{\beta}(f(y), f(z)) \leq \frac{\left(1+k_{\beta}\right)}{2} d_{\beta}(y, z)
$$

and hence $y=z$.

In the case where $E=(E, d)$ is a hyperconvex metric space, and $F_{\alpha}$ has closed bounded values, then (iii) can be replaced by

(iii) $^{\prime} \forall \varepsilon>0 \exists \alpha \in \Lambda$ such that $\operatorname{diam}\left(F_{\alpha}(x)\right)<\varepsilon \quad \forall x \in E$.

Recall that $E$ is hyperconvex if for every $X$ metric space, $Y \subset X$, and $g: Y \rightarrow E$ non-expansive, there exists $\hat{g}: X \rightarrow E$ a non-expansive extension of $g$.

Let us mention that Espínola and Kirk's results were recently generalized by Espínola and Petruşel [18].

6. Multi-valued contractions in gauge spaces. In this section, we present two fixed point results for multi-valued contractions in gauge spaces obtained by Frigon [21]. As in the previous section, $\left(E,\left\{d_{\alpha}\right\}_{\alpha \in \Lambda}\right)$ is a complete gauge space but we do not assume that $\Lambda$ is a directed set. 
Definition 6.1. Let $X \subset E$. A multi-valued map $F: X \rightarrow E$ is called an admissible contraction with constant $k=\left\{k_{\alpha}\right\}_{\alpha \in \Lambda} \in[0,1)^{\Lambda}$ if

(i) for every $\alpha \in \Lambda, D_{\alpha}(F(x), F(y)) \leq k_{\alpha} d_{\alpha}(x, y)$ for every $x, y \in X$;

(ii) for every $x \in X$ and every $\varepsilon \in(0, \infty)^{\Lambda}$, there exists $y \in F(x)$ such that $d_{\alpha}(x, y) \leq$ $\operatorname{dist}_{\alpha}(x, F(x))+\varepsilon_{\alpha}$ for every $\alpha \in \Lambda$.

Observe that if $\Lambda=\mathbb{N}, E$ is metrizable with some metric $d$. However, a multi-valued map $F$ can be an admissible contraction without being a contraction in the usual sense when $E$ is endowed with the metric $d$.

We start with a generalization of Nadler's fixed point theorem (Theorem 3.1) and of Cain and Nashed's result (Theorem 5.1).

THEOREM 6.2. Let $E$ be a complete gauge space, and $F: E \rightarrow E$ an admissible multivalued contraction with closed values. Then $F$ has a fixed point.

Proof. Let $k \in[0,1)^{\Lambda}$ be a constant of contraction of $F$. Fix $x_{0} \in E$. For every $\alpha \in \Lambda$, choose $r_{\alpha}>0$ such that $\operatorname{dist}_{\alpha}\left(x_{0}, F\left(x_{0}\right)\right)<\left(1-k_{\alpha}\right) r_{\alpha}$. We can choose $x_{1} \in F\left(x_{0}\right)$ such that

$$
d_{\alpha}\left(x_{1}, x_{0}\right)<\left(1-k_{\alpha}\right) r_{\alpha} \text { for every } \alpha \in \Lambda .
$$

Then, choose $x_{2} \in F\left(x_{1}\right)$ such that for every $\alpha \in \Lambda$,

$$
\begin{aligned}
d_{\alpha}\left(x_{1}, x_{2}\right) & <\operatorname{dist}_{\alpha}\left(x_{1}, F\left(x_{1}\right)\right)+k_{\alpha}\left(\left(1-k_{\alpha}\right) r_{\alpha}-d_{\alpha}\left(x_{0}, x_{1}\right)\right) \\
& \leq D_{\alpha}\left(F\left(x_{0}\right), F\left(x_{1}\right)\right)+k_{\alpha}\left(\left(1-k_{\alpha}\right) r_{\alpha}-d_{\alpha}\left(x_{0}, x_{1}\right)\right) \\
& \leq k_{\alpha} d_{\alpha}\left(x_{0}, x_{1}\right)+k_{\alpha}\left(\left(1-k_{\alpha}\right) r_{\alpha}-d_{\alpha}\left(x_{0}, x_{1}\right)\right) \\
& =k_{\alpha}\left(1-k_{\alpha}\right) r_{\alpha} .
\end{aligned}
$$

Repeating this process permits to obtain a sequence $\left\{x_{n}\right\}$ such that

$$
d_{\alpha}\left(x_{n}, x_{n+1}\right)<k_{\alpha}^{n}\left(1-k_{\alpha}\right) r_{\alpha} \quad \text { for every } \alpha \in \Lambda \text {. }
$$

Therefore, $\left\{x_{n}\right\}$ is a Cauchy sequence and hence converges to some $x$. The continuity of $F$ implies that $x \in F(x)$.

Here is a continuation result on a closed subset which can have empty interior.

TheOrem 6.3. Let $E$ be a complete gauge space, $X \subset E$ closed, and $H: X \times[0,1] \rightarrow E$ be a multi-valued map with closed values. Assume that

(i) there exists $k \in[0,1)^{\Lambda}$ such that for every $t \in[0,1], H(\cdot, t)$ is an admissible contraction with constant $k$;

(ii) for every $\alpha \in \Lambda$, there exists $M_{\alpha}<1$ such that $D_{\alpha}(H(x, t), H(x, s)) \leq M_{\alpha}|t-s|$ for every $s, t \in[0,1]$ and every $x \in X$;

(iii) $x \notin H(x, t)$ for all $t \in[0,1]$ and $x \in X_{k, M}$ where

$$
\begin{aligned}
& X_{k, M}=\left\{y \in X: \bigcap_{\alpha} B_{\alpha}\left(y, r_{\alpha}\right) \not \subset X \text { for every } r=\left(r_{\alpha}\right)_{\alpha \in \Lambda} \in[0, \infty)^{\Lambda}\right. \\
& \left.\quad \text { with } \inf _{\alpha} \frac{r_{\alpha}\left(1-k_{\alpha}\right)}{M_{\alpha}}>0\right\} .
\end{aligned}
$$

If $H(\cdot, 0)$ has a fixed point, then $H(\cdot, t)$ has a fixed point for all $t \in[0,1]$. 
Proof. The argument is similar to the proof of Theorem 4.1. Consider

$$
Q=\{(x, t) \in X \times[0,1]: x \in H(x, t)\} .
$$

We define on $Q$ the partial order

$$
(x, t) \leq(y, s) \text { if and only if } t \leq s \text { and } d_{\alpha}(x, y) \leq \frac{2 M_{\alpha}(s-t)}{1-k_{\alpha}} \text { for every } \alpha \in \Lambda .
$$

It is easy to show that every totally ordered subset of $Q$ has an upper bound.

From Zorn's Lemma, $Q$ has a maximal element $\left(x_{0}, t_{0}\right) \in Q$, so $x_{0} \in H\left(x_{0}, t_{0}\right)$. To conclude, we need to show that $t_{0}=1$. If this is false, since $x_{0} \notin X_{k, M}$, there exist $r \in[0, \infty)^{\Lambda}$ and $t_{1} \in\left(t_{0}, 1\right]$ such that

$$
B:=\bigcap_{\alpha \in \Lambda} B_{\alpha}\left(x_{0}, r_{\alpha}\right) \subset X \quad \text { and } \quad \frac{2 M_{\alpha}\left(t_{1}-t_{0}\right)}{1-k_{\alpha}}=r_{\alpha} \quad \text { for every } \alpha \in \Lambda .
$$

On the other hand, for every $\alpha \in \Lambda$,

$$
\begin{aligned}
\operatorname{dist}_{\alpha}\left(x_{0}, H\left(x_{0}, t_{1}\right)\right) & \leq \operatorname{dist}_{\alpha}\left(x_{0}, H\left(x_{0}, t_{0}\right)\right)+D_{\alpha}\left(H\left(x_{0}, t_{0}\right), H\left(x_{0}, t_{1}\right)\right) \\
& \leq M_{\alpha}\left(t_{1}-t_{0}\right)<\left(1-k_{\alpha}\right) r_{\alpha} .
\end{aligned}
$$

Arguing as in Theorem 6.3 , there exists $x_{1} \in B$ a fixed point of $H\left(\cdot, t_{1}\right)$. So, $\left(x_{1}, t_{1}\right) \in Q$ and $\left(x_{0}, t_{0}\right)<\left(x_{1}, t_{1}\right)$, which is a contradiction.

In the previous theorem, if $X=\bar{U}$ with $U$ open, $U_{k, M}=\partial U$ and hence, (iii) can be replaced by

(iii) $)^{\prime} \quad x \notin H(x, t)$ for all $x \in \partial U$ and all $t$.

Agarwal, Cho and O'Regan [1] generalized this result in the particular case of $U$ an open subset of $E$ and for $H: \bar{U} \times[0,1] \rightarrow E$ satisfying a more general condition of contraction. Condition (iii) was also weakened.

Acknowledgments. The author would like to thank the referee for his careful reading of the manuscript, and for suggestions and remarks that improved the final outcome.

\section{References}

[1] R. Agarwal, Y. J. Cho. D. O'Regan, Homotopy invariant results on complete gauge spaces, Bull. Austral. Math. Soc. 67 (2003), 241-248.

[2] R. Agarwal, D. O'Regan, The homotopic invariance for fixed points and fuzzy fixed points of multivalued generalized contractive maps, Nonlinear Stud. 10 (2003), 187-193.

[3] N. A. Assad, W. A. Kirk, Fixed point theorems for set-valued mappings of contractive type, Pacific J. Math. 43 (1972), 553-562.

[4] S. Banach, Sur les opérations dans les ensembles abstraits et leurs applications aux équations intégrales, Fund. Math. 3 (1922), 133-181.

[5] F. E. Bonsall, Lectures on Some Fixed Point Theorems of Functional Analysis, Tata Institute of Fundamental Research, Bombay 1962.

[6] D. W. Boyd, J. S. W. Wong, On nonlinear contractions, Proc. Amer. Math. Soc. 20 (1969), 458-464.

[7] F. Browder, On the convergence of successive approximations for nonlinear functional equations, Nederl. Akad. Wetensch. Proc. Ser. A 71=Indag. Math. 30 (1968), 27-35. 
[8] R. Caccioppoli, Una teorema generale sull'esistenza di elementi uniti in una transformazione funzionale, Rend. Acad. Naz. Lincei 11 (1930), 794-799.

[9] G. L. Cain, Jr., M. Z. Nashed, Fixed points and stability for a sum of two operators in locally convex spaces, Pacific J. Math. 39 (1971), 581-592.

[10] J. Caristi, Fixed points theorems for mappings satisfying inwardness conditions, Trans. Amer. Math. Soc. 215 (1976), 241-251.

[11] A. Chiş, R. Precup, Continuation theory for general contractions in gauge spaces, Fixed Point Theory Appl. 3 (2004), 173-185.

[12] H. Covitz, S. B. Nadler, Jr., Multi-valued contraction mappings in generalized metric spaces, Israel J. Math. 8 (1970), 5-11.

[13] J. Dugundji, Topology, Allyn and Bacon, Boston 1966.

[14] J. Dugundji, A. Granas, Weakly contractive maps and elementary domain invariance theorem, Bull. Greek Math. Soc. 19 (1978), 141-151.

[15] M. Edelstein, An extension of Banach's contraction principle, Proc. Amer. Math. Soc. 12 (1961), 7-10.

[16] M. Edelstein, On fixed and periodic points under contractive mappings, J. London Math. Soc. 37 (1962), 74-79.

[17] R. Espínola, W. A. Kirk, Set-valued contractions and fixed points, Nonlinear Anal. 54 (2003), 485-494.

[18] R. Espínola, A. Petruşel, Existence and data dependence of fixed points for multivalued operators on gauge spaces, J. Math. Anal. Appl. 309 (2005), 420-432.

[19] M. Frigon, On continuation methods for contractive and nonexpansive mappings, in: Recent Advances on Metric Fixed Point Theory (Seville, 1995), Ciencias 48, Univ. Sevilla, Seville 1996, 19-30.

[20] M. Frigon, Fixed point results for generalized contractions in gauge spaces and applications, Proc. Amer. Math. Soc. 128 (2000), 2957-2965.

[21] M. Frigon, Fixed point results for multivalued contractions on gauge spaces, in: Set Valued Mappings with Applications in Nonlinear Analysis, Ser. Math. Anal. Appl. 4, Taylor \& Francis, London 2002, 175-181.

[22] M. Frigon, A. Granas, Résultats de type Leray-Schauder pour des contractions multivoques, Topol. Methods Nonlinear Anal. 4 (1994), 197-208.

[23] M. Frigon, A. Granas, Résultats de type Leray-Schauder pour des contractions sur des espaces de Fréchet, Ann. Sci. Math. Québec 22 (1998), 161-168.

[24] M. Frigon, A. Granas, Z. E. A. Guennoun, Alternative non linéaire pour les applications contractantes, Ann. Sci. Math. Québec 19 (1995), 65-68.

[25] M. Frigon, D. O'Regan, Fuzzy contractive maps and fuzzy fixed points, Fuzzy Sets Systems 129 (2002), 39-45.

[26] J. A. Gatica, W. A. Kirk, Fixed point theorems for contraction mappings with applications to nonexpansive and pseudo-contractive mappings, Rocky Mountain J. Math. 4 (1974), 69-79.

[27] M. A. Geraghty, On contractive mappings, Proc. Amer. Math. Soc. 40 (1973), 604-608.

[28] N. Gheorghiu, Fixed point theorems in uniform spaces, An. Ştiinţ. Univ. "Al. I. Cuza" Iaşi Secţ. I a Mat. 28 (1982), 17-18.

[29] K. Goebel, W. A. Kirk, Topics in Metric Fixed Point Theory, Cambridge Stud. Adv. Math. 28, Cambridge Univ. Press, Cambridge 1990.

[30] K. Goebel, S. Reich, Uniform Convexity, Hyperbolic Geometry, and Nonexpansive Mappings, Marcel Dekker, New York 1984. 
[31] A. Granas, Continuation methods for contractive maps, Topol. Methods Nonlinear Anal. 3 (1994), 375-379.

[32] A. Granas, J. Dugundji, Fixed Point Theory, Springer Monogr. Math., Springer, New York 2003.

[33] B. R. Halpern, G. M. Bergman, A fixed point theorem for inward and outward maps, Trans. Amer. Math. Soc. 130 (1968), 353-358.

[34] J. Jachymski, Continuation dependence of attractors of iterated function systems, J. Math. Anal. Appl. 198 (1996), 221-226.

[35] J. Jachymski, Equivalence of some contractivity properties over metrical structures, Proc. Amer. Math. Soc. 125 (1997), 2327-2335.

[36] M. A. Khamsi, W. A. Kirk, An Introduction to Metric Spaces and Fixed Point Theory, Pure Appl. Math. (N.Y.), Wiley-Interscience, New York 2001.

[37] W. A. Kirk, Contraction mappings and extensions, in: Handbook of Metric Fixed Point Theory, Kluwer, Dordrecht 2001, 1-34.

[38] R. J. Knill, Fixed points of uniform contractions, J. Math. Anal. Appl. 12 (1965), 449-455.

[39] T. C. Lim, On fixed point stability for set-valued mappings with applications to generalized differential equations, J. Math. Anal. Appl. 110 (1985), 436-441.

[40] J. Matkowski, Nonlinear contractions in metrically convex spaces, Publ. Math. Debrecen 45 (1994), 103-114.

[41] N. Mizoguchi, W. Takahashi, Fixed point theorems for multivalued mappings on complete metric spaces, J. Math. Anal. Appl. 141 (1989), 177-188.

[42] S. B. Nadler, Jr., Sequences of contractions and fixed points, Pacific J. Math. 27 (1968), $579-585$.

[43] S. B. Nadler, Jr., Multi-valued contraction mappings, Pacific J. Math. 30 (1969), 475-488.

[44] S. B. Nadler, Jr., Periodic points of multi-valued $\varepsilon$-contractive maps, Topol. Methods Nonlinear Anal. 22 (2003), 399-409.

[45] R. Nussbaum, The fixed point index and asymptotic fixed point theorems for $k$-set contractions, Ph.D. Thesis, University of Chicago 1969.

[46] D. O'Regan, R. Precup, Continuation theory for contractions on spaces with two vectorvalued metrics, Applicable Anal. 82 (2003), 131-144.

[47] J. Polewczak, Ordinary differential equations on closed subsets of Fréchet spaces with applications to fixed point theorems, Proc. Amer. Math. Soc. 107 (1989), 1005-1012.

[48] R. Precup, Discrete continuation method for boundary value problems on bounded sets in Banach spaces, J. Comput. Appl. Math. 113 (2000), 267-281.

[49] R. Precup, Continuation results for mappings of contractive type, Semin. Fixed Point Theory Cluj-Napoca 2 (2001), 23-40.

[50] E. Rakotch, A note on contractive mappings, Proc. Amer. Math. Soc. 13 (1962), 459-465.

[51] S. Reich, Some remarks concerning contraction mappings, Canad. Math. Bull. 14 (1971), 121-124.

[52] S. Reich, Kannan's fixed point theorem, Boll. Un. Mat. Ital. (4) 4 (1971), 1-11.

[53] S. Reich, A fixed point theorem for locally contractive multi-valued functions, Rev. Roumaine Math. Pures Appl. 17 (1972), 569-572.

[54] S. Reich, Fixed points of contractive functions, Boll. Un. Mat. Ital. (4) 5 (1972), 26-42.

[55] S. Reich, On fixed point theorems obtained from existence theorems for differential equations, J. Math. Anal. Appl. 54 (1976), 26-36. 
[56] S. Reich, Approximate selections, best approximations, fixed points, and invariant sets, J. Math. Anal. Appl. 62 (1978), 104-113.

[57] S. Reich, A fixed point theorem for Fréchet spaces, J. Math. Anal. Appl. 78 (1980), 33-35.

[58] S. Reich, A. J. Zaslavski, Almost all nonexpansive mappings are contractive, C. R. Math. Acad. Sci. Soc. R. Can. 22 (2000), 118-124.

[59] S. Reich, A. J. Zaslavski, The set of noncontractive mappings is $\sigma$-porous in the space of all nonexpansive mappings, C. R. Acad. Sci. Paris Sér. I Math. 333 (2001), 539-544.

[60] B. E. Rhoades, A comparison of various definitions of contractive mappings, Trans. Amer. Math. Soc. 226 (1977), 257-290.

[61] I. A. Rus, A. Petruşel, A. Sîntămărian, Data dependence of the fixed point set of some multivalued weakly Picard operators, Nonlinear Anal. 52 (2003), 1947-1959.

[62] E. Tarafdar, An approach to fixed point theorems on uniform spaces, Trans. Amer. Math. Soc. 191 (1974), 209-225.

[63] J. Weissinger, Zur Theorie und Anwendung des Iterationsverfahrens, Math. Nachr. 8 (1952), 193-212. 